\title{
The expression of diacylglycerol kinase theta during the organogenesis of mouse embryos
}

\author{
Shuji Ueda', Becky Tu-Sekine², Minoru Yamanoue', Daniel M Raben² and Yasuhito Shirai ${ }^{\text {* }}$
}

\begin{abstract}
Background: Diacylglycerol kinase (DGK) is a key enzyme that regulates diacylglycerol (DG) turnover and is involved in a variety of physiological functions. The isoform DGK $\theta$ has a unique domain structure and is the sole member of type $V$ DGK. To reveal the spatial and temporal expression of DGK $\theta$ we performed immunohistochemical staining on paraffin sections of mouse embryos.

Results: At an early stage of development (E10.5 and 11.5), the expression of DGK $\theta$ was prominently detected in the brain, spinal cord, dorsal root ganglion, and limb bud, and was also moderately detected in the bulbus cordis and the primordium of the liver and gut. At later stages (E12.5 and 14.5), DGK $\theta$ expression persisted or increased in the neocortex, epithalamus, hypothalamus, medulla oblongata, and pons. DGK $\theta$ was also evident in the epidermis, and nearly all epithelia of the oropharyngeal membrane, digestive tract, and bronchea. At prenatal developmental stages (E16.5 and E18.5), the expression pattern of DGKO was maintained in the central nervous system, intestine, and kidney, but was attenuated in the differentiated epidermis.

Conclusion: These results suggest that DGK $\theta$ may play important physiological roles not only in the brain, but also in diverse organs and tissues during the embryonic stages.
\end{abstract}

Keywords: Diacylglycerol kinase, Embryonic development, Central nerve system, Epidermis

\section{Background}

Many hormones and growth factors stimulate phospholipase $C$ by activating its receptor. This activation results in the production of diacylglycerol (DG) at the plasma membrane, which triggers the activation of several enzymes, such as chimerins [1], Ras guanyl nucleotide-releasing protein (RasGRPs) [2,3], transient receptor potential cation channel C (TRPC) [4], and both conventional and novel PKCs [5,6]. DG kinase (DGK) phosphorylates DG to produce phosphatidic acid (PA), resulting in the inhibition of DG-mediated intracellular signal transduction. Additionally, $\mathrm{PA}$ is a lipid second messenger that regulates various target proteins, including atypical PKC, mTOR, and phosphatidylinositol 4-phosphate 5-kinase [7,8]. Thus, DGK plays pivotal roles in various intracellular signaling pathways by regulating the DG and PA levels [9-12]. Ten DGK isoforms have been identified in mammal, and classified

\footnotetext{
* Correspondence: shirai@kobe-u.ac.jp

${ }^{1}$ Department of Agrobioscience, Graduate School of Agricultural Science,

Kobe University, Kobe, Hyogo, Japan

Full list of author information is available at the end of the article
}

into five subtypes, from I to $\mathrm{V}$, based on similarities in the domain structures.

The physiologic roles of DGK isoforms have been partially elucidated by analyses of DGK gene expression patterns and of genetically modified organisms. For example, DGK $\beta$ is a major isoform expressed in the brain, and the disruption of this isoform impairs memory and causes antidepressant-like effects in mice [13]. In contrast, DGK $\alpha$ enhances interleukin 2-induced $\mathrm{T}$ cell proliferation [14], and knockout $(\mathrm{KO})$ mice exhibit impaired induction of $\mathrm{T}$ cell anergy [15]. Another isoform, DGK $\zeta$ is a type IV DGK that is ubiquitously expressed in most mouse tissues. DGK $\zeta$ $\mathrm{KO}$ mice exhibit abnormalities in multiple tissues, including a decrease in the number of dendritic spines, and an impairment of the immune response $[4,16,17]$. In regard to nervous system, a large number of studies have shown the roles of DGKs in neuronal spine density, synaptic activity, epileptogenesis and neuronal plasticity in mammals $[18,19]$ and C. elegans used as a genetic model [20]. It is not clear the relationships of ten DGK isoforms in mammal. To better understand the role of each DGK and its redundancy
C Biomed Central

(c) 2013 Ueda et al.; licensee BioMed Central Ltd. This is an Open Access article distributed under the terms of the Creative Commons Attribution License (http://creativecommons.org/licenses/by/2.0), which permits unrestricted use, distribution, and reproduction in any medium, provided the original work is properly cited. 
among the DGK family, further studies in other subtypes of DGKs are necessary.

DGK $\theta$ was originally cloned from the rat brain and identified as the sole type V DGK [21]. DGK $\theta$ contains three C1 domains and a Ras-association (RA) domain in the central region. Studies have shown that DGK $\theta$ is enriched in the nuclear matrix of various cultured cell lines [22], is negatively regulated by its interaction with the small GTPase RhoA [23], and translocate to the plasma membrane following phosphorylation by $\mathrm{PKC} \varepsilon$ [24]. The optimal activation of DGK $\theta$ may require both polybasic protein and acidic phospholipid cofactors, which have been shown to stimulate DGK $\theta$ synergistically in vitro $[25,26]$. These multiple regulatory mechanisms enable DGK $\theta$ to mediate signals from a wide variety of extracellular ligands [24,27] including epidermal growth factor [28], nerve growth factor [29], $\alpha$-thrombin [30], and adenosine[31].

DGK $\theta$ is highly expressed in rat brain [21] and is present in a variety of cultured cell lines [22,24]. However, there is limited information regarding this enzyme's cell type-specific functions or its physiological roles in mammals. In this study, we examined the distribution and changes in expression from E10 to E17 to reveal the spatial and temporal expression of DGK $\theta$ protein in mouse embryos.

\section{Results}

\section{Expression patterns of DGK $\theta$ in E10.5 to E16.5 mouse embryos}

To determine the distribution of DGK $\theta$, we performed immunohistochemistry (IHC) on paraffin sections of mouse embryos. We used two individual antibodies that target the C-terminal region of the protein, anti-DGK $\theta$ antibodies \#1 and \#2, to validate immunostaining patterns. Both antibodies detected a major 110-kDa band of DGK $\theta$ on an immunoblotting membrane containing the extract from whole brain (Additional file 1: Figure S1 C), in which DGK $\theta$ had been detected with RT-PCR (data not shown). The observed molecular size coincided with the size of endogenous protein $(110 \mathrm{kDa})$ in HEK293 and HeLa cell lysates by immunoblotting $[16,23]$. The specificity of this antibody was further confirmed by the transient transfection. These antibodies successfully detected exogenously expressed recombinant DGK $\theta$ protein by immunofluorescence in HeLa cells, but not other subtypes (Additional file 2: Figure S2 and data not shown).

At E10.5, the immunoreactivity of DGK $\theta$ was noticeable along the surface of the forebrain $(\mathrm{fb})$, rhombencephalon (rb), and neural tube (nt) (Figure 1A-C and F), and was also distinctly observable in the ectodermal epithelium of the hind bud (hb) (Figure 1H) (evident as brown staining in all images). DGK $\theta$ expression was also detected in the branchial arch (ba), bulbus cordis (bc), hepatic primordium (hp), midgut artery (ma), and notal cord (nc) (Figure 1A). A similar staining pattern persisted at E11.5. Immunoreactivity of DGK $\theta$ was detected in the bulbus cordis, liver (li), and lumen of the stomach and midgut (mg) (Figure 1I and M). In addition to the distribution observed at E10.5, DGK $\theta$ staining was noted in the tongue (to), nasal placode (np), primitive bronchi (br), the dorsal root ganglion ( $\mathrm{drg}$ ), and the trachea (tr) separating from the esophagus at E11.5 (Figure $1 \mathrm{I}, \mathrm{K}$ and $\mathrm{L}$ ). It is noteworthy that the immunoreactivity significantly increased in the neuroepithelium surrounding the third ventricle (tv) at E11.5 compared with that at E10.5 (Figure 1K). In contrast to the faint staining of the mesenchymal cells around the cephalic region, the immunoreactivity of the mesenchymal cells around the peripheries of the tongue (to) and nasal placode $(n p)$ was relatively high (Figure $1 \mathrm{~A}-\mathrm{B}$ and I).

The embryonic brain grows rapidly until E12.5, and the forebrain partly divides into the telencephalon $(\mathrm{t})$ and diencephalon (d). Simultaneously, a pharynx membrane and respiratory apparatus rapidly develop. In the current study, at E12.5 the immunoreactivity in the cephalic region increased intensively at the rostral side of the telencephalon, the tectum of midbrain, and the pontine flexure (pf) in which neuroepithelial cells proliferate (Figure 2A-C). Furthermore, the immunoreactivity persisted or increased in multiple organs (tongue, lung, liver, heart, and midgut) (Figure $2 \mathrm{~A}$ and $\mathrm{D}$ ), and expanded to the surface layer of the nasal cavity and dental lamina (dl) (Figure 2E). A similar pattern of distribution was observed at E13.5 (data not shown).

In the developing central nervous system region at E14.5, DGK $\theta$ immunoreactivity was widely distributed in all brain regions. Particularly dense immunoreactivity was observed in the neocortex (nx), epithalamus (epi), medulla oblongata (mo), and the gray horn of spinal cord (Figure 2F-I, K and $\mathrm{N}$ ). However, DGK $\theta$ was not detected in the ganglionic eminence (ge), which localizes to the medial side of the lateral ventricle (Figure $2 \mathrm{H}$ ). In other areas, dense immunoreactivity was continually detected within various types of epithelia, including the tongue (to), tooth bud (tb), nasal cavity (nc), bronchus (br), midgut (mg), and urogenital sinus (us) (Figure 2F, I, J, M, O and P). In contrast to the immunoreactivity predominantly observed in the epithelia classified as simple or stratified epithelium, DGK $\theta$ protein was undetectable in the endothelial cells of the arterial trunk (Figure 2A and F). Beginning at E14.5, the fetal skin increased in thickness and formed the layer of the squamous epithelium. At the facial and dorsal surfaces we found a significant increase of immunoreactivity in the squamous epithelial cells, which successively differentiate into epidermis (Figure 2J and N). IHC without the DGKO antibody failed to detect any signal in the sections from E10.5 to E14.5 (data not shown). 

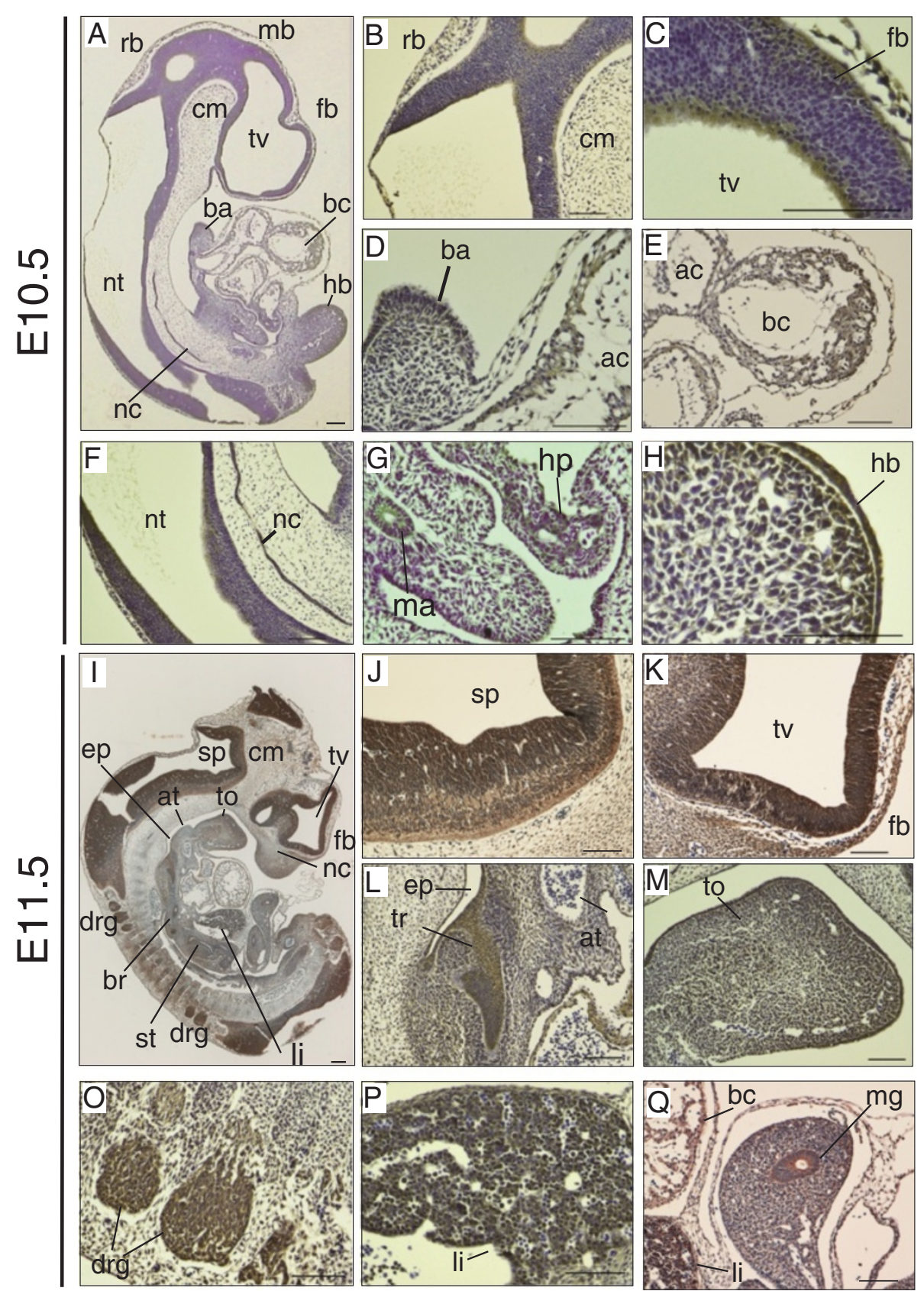

Figure 1 Expression patterns of DGK $\theta$ at E10.5 and E11.5. (A, I) Sagittal sections showing the distribution of DGK $\theta$. (B-H) High magnification images showing DGKO-positive regions at E10.5. Forebrain ( $\mathrm{fb}$ ), midbrain (mb), rhombencephalon (rb), third ventricle (tv), cephalic mesenchyme (cm), branchial arch (ba), atrial chamber (ac), bulbus cordis (bc), notochord (nc), hepatic primordium (hp), midgut artery (ma), hindlimb bud (hb). (J-Q) High magnification images showing DGKO positive region at E11.5. Spinal cord (sp), esophagus (ep), nasal placode (np), trachea (tr), tongue (to), bronchi (br), stomach (st), liver (li), arterial trunk aortic arch (aa), dorsal root ganglion (drg), midgut (mg). The sections were stained with antiDGK $\theta$ antibody \#1. The scale bars show $100 \mu \mathrm{m}$.

\section{Expression patterns of DGKO in late stages of embryogenesis}

At E16.5, the immunoreactivity of DGK $\theta$ was maintained in the neocortex (nx) and the mesencephalon (Figure 3A), and increased in the villi (vi) and mucosa $(\mathrm{mu})$ of the intestine (Figure $3 \mathrm{C}$ ) and the basal layer (bl) of the dorsal skin (ds) (Figure 3D). In contrast, we found that the immunoreactivity of DGK $\theta$ was attenuated in the lung (Figure 3B) and liver at E16.5. At prenatal developmental stages (E17.5 and E18.5), DGK $\theta$ immunoreactivity was unchanged in the brain region relative to the E14.5. In other areas, immunoreactivity was faintly observed in the enamel epithelium of 

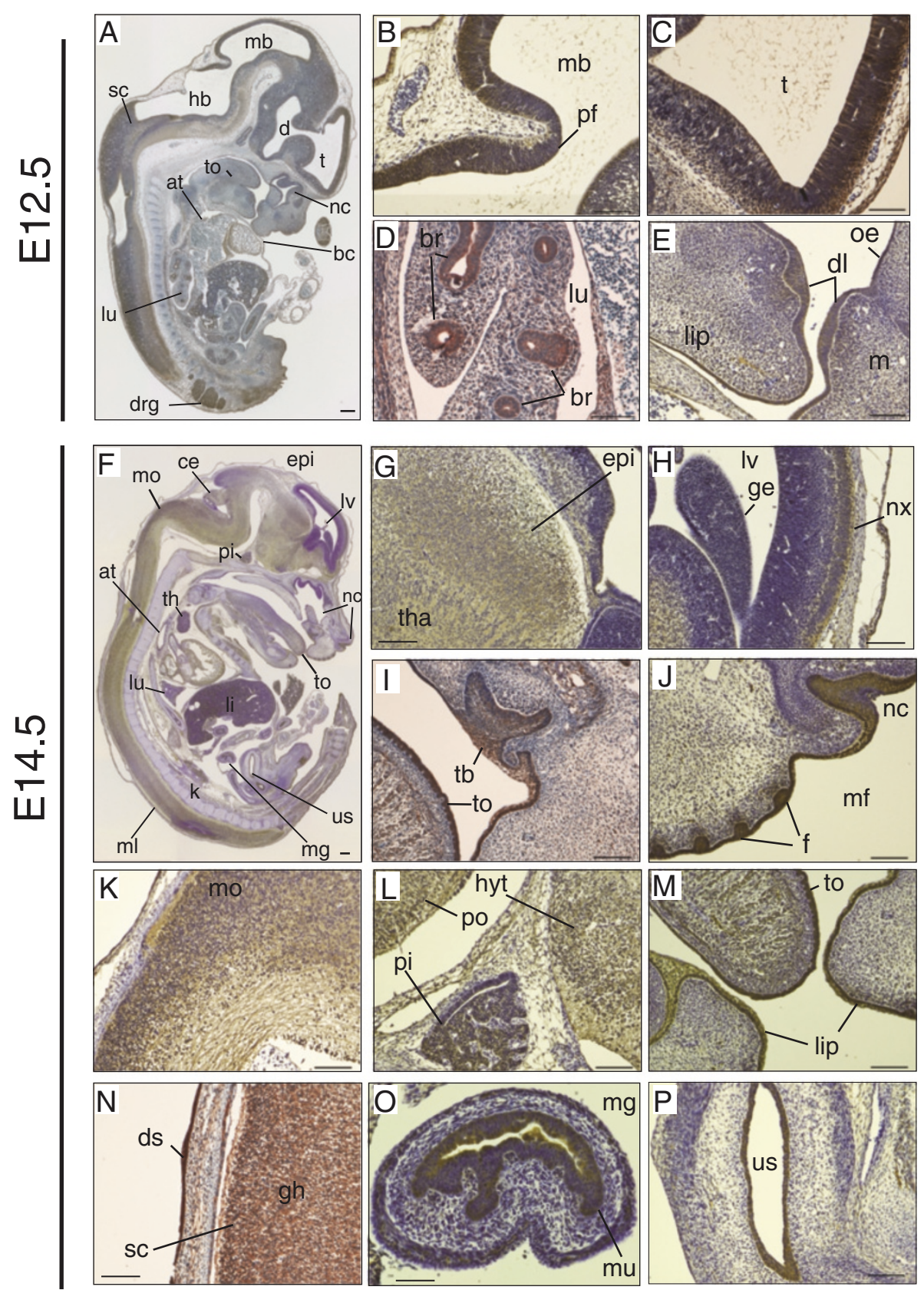

Figure 2 Expression patterns of DGK $\theta$ at E12.5 and E14.5. (A, F) Sagittal sections showing the distribution of DGK . (B-E) High-magnification images showing DGKO-positive regions at E12.5. Telencephalon (t), diencephalon (d), midbrain (mb), arterial trunk (at), tongue (to), lung (lu), dorsal root ganglion (drg), bronchus (br), dental lamina (dl), oral epithelium (oe). (F-P) High-magnification images showing DGK $\theta$-positive regions at E14.5. Neocortex (nx), lateral ventricle (Iv), ganglionic eminence (ge), epithalamus (epi), cerebellum (ce), medulla oblongata (mo), pituitary gland (pi), pons (po), hypothalamus (hyt), nasal cavity (nc), tooth bud (tb), follicle (f), lung (lu), liver (li), dorsal skin (dr), midgut (mg), mucosa layer (mu), urogenital sinus (us), kidney (k). The sections were stained with anti-DGK $\theta$ antibody \#1. The scale bars indicate $100 \mu \mathrm{m}$.

incisor teeth (Figure 3E), the epidermis of the facial and dorsal surfaces (Figure $3 \mathrm{H}$ and $\mathrm{K}$ ), the walls of the tranche and main bronchus, and the ventricular wall of the heart (Figure 3I and J), while strong signals persisted in the villi and mucosa of the intestine (Figure $3 \mathrm{M}$ ) and in the collecting tubule (ct) and Bowman's capsule (bc) of the kidney (Additional file 3: Figure S3 A and D). The intensity and pattern of the immunoreactivity was also verified by examining serial sections with two different anti-DGK $\theta$ (antibodies \#1 and \#2) (Additional file 3: Figure S3 B and C). When the anti-DGK $\theta$ antibody was preincubated with the blocking polypeptide prior to IHC, no immunostaining 

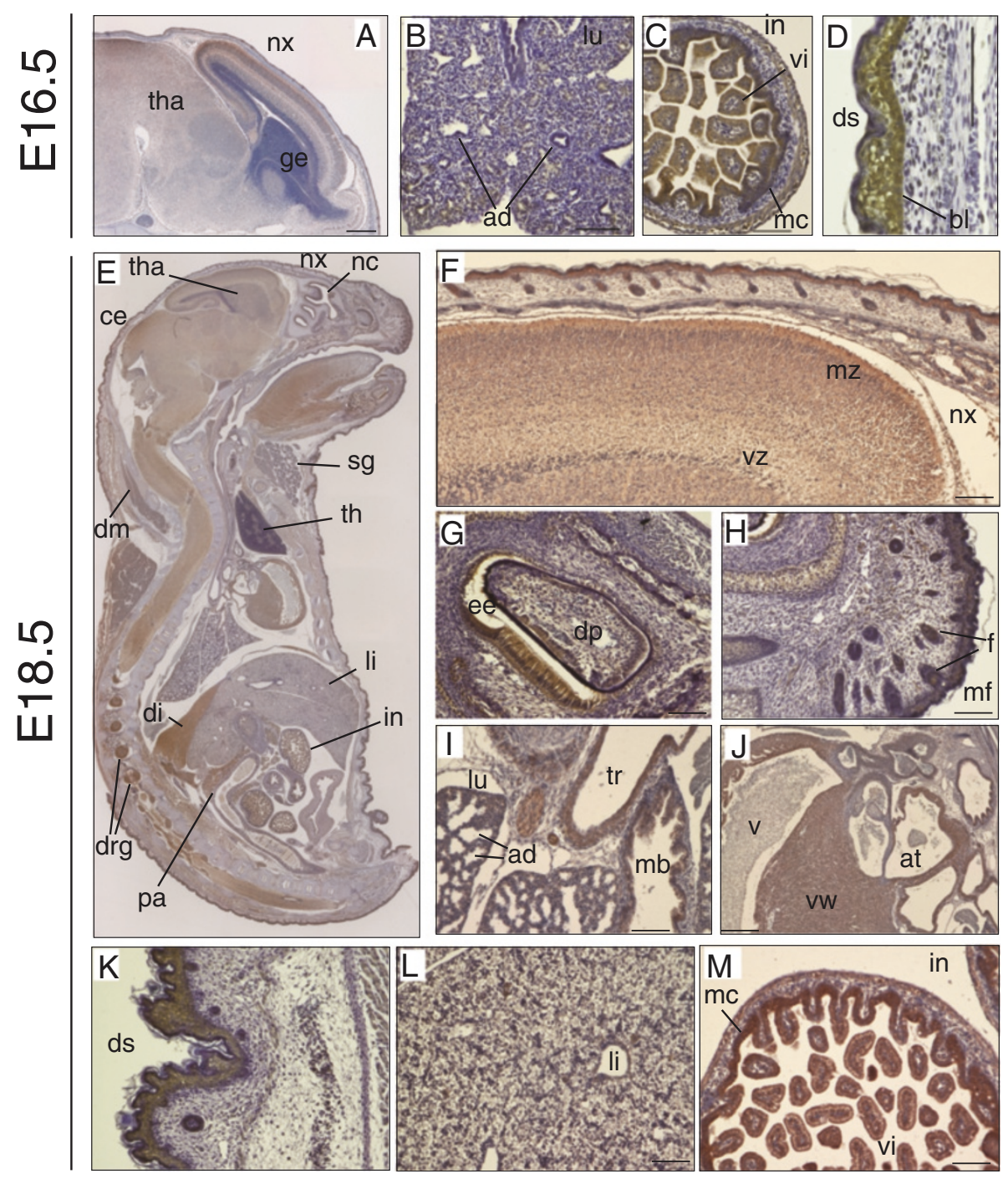

Figure 3 Expression patterns of DGK $\theta$ at E16.5 and E18.5. (A, E) Sagittal sections showing the distribution of DGKO. (B-D) High-magnification images showing discriminative regions at E16.5. Thalamus (tha), neocortex (nx), ganglionic eminence (ge), lung (lu), alveolar duct (ad), intestine (in), villi (vi), mucosa (mc), dorsal skin (ds), basal layer (bl). (E-M) High magnification images showing discriminative regions at E18.5. cerebellum (ce), salivary glands ( $\mathrm{sg}$ ), thymus (th), dorsal muscles (dm), diaphragm (di), dorsal root ganglion (drg), pancreas (pa), marginal zone (mz),

ventricular zone (vz), dental pulp (dp), enamel epithelium (ee), follicle ( $f$ ), muffle (mf), throat (tr), aortic trunk (at), ventricle (v), ventricular wall ( $\mathrm{vw}$ ) The sections were stained with anti-DGKO antibody \#1. The scale bars indicate $100 \mu \mathrm{m}$.

was detected at E17.5 with the exception of the pancreas (pa) and the surface of the villi in the duodenum, which corresponded with IHC results obtained without the primary antibody (Additional file 3: Figure S3 D and E).

\section{RT-PCR analysis of DGK $\theta$ expression in mouse embryos}

To confirm the distribution of DGK $\theta$, we analyzed the expression of the DGK $\theta$ mRNA at E14.5 using semiquantitative RT-PCR. The results demonstrated that DGK $\theta$ was expressed in multiple organs (brain, lung, liver, intestine, kidney, and dorsal skin) at E14.5. Additionally, DGK $\theta$ was expressed more abundantly in the brain and dorsal skin than in the other organs (Figure 4A). This expression profile of the mRNA is consistent with the immunohistochemical observation, which indicated that DGK $\theta$ was distributed among various epithelia and neurons at E14.5.

To examine the change in DGK $\theta$ expression during development, we analyzed the expression in dorsal skin from E14.5 until adulthood. In the developmental skin, the expression of DGK $\theta$ was significantly higher at E14.5 than at E17.5 and during adulthood (Figure 4A and B).

\section{Discussion}

To examine the regional distribution of DGK $\theta$ during the embryonic period, we performed immunohistochemistry (IHC) on whole mouse embryos. The immunostaining 


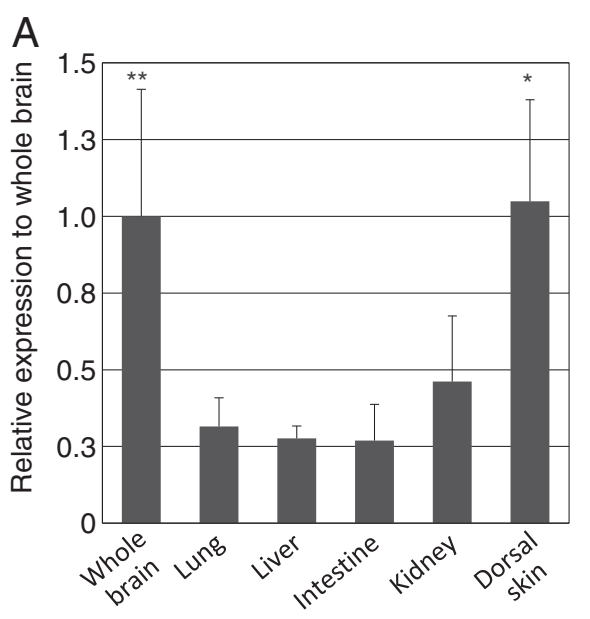

At E14.5

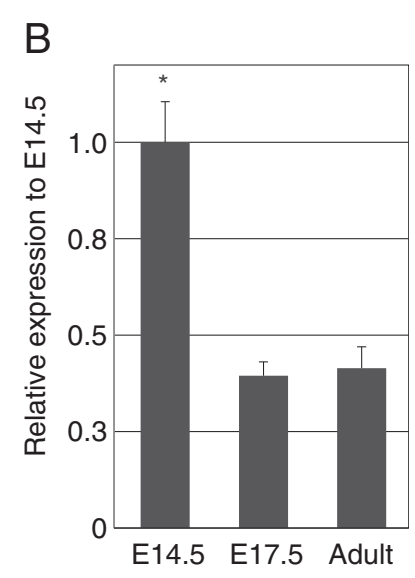

\section{Dorsal skin}

Figure 4 RT-PCR analysis of DGK $\boldsymbol{\theta}$ expression in mouse embryo. The DGK $\theta$ mRNA levels were determined by semiquantitative RT-PCR. The number of amplification cycles for DGK $\theta$ and GAPDH were 35 and 28, respectively. The amount of template for each PCR was normalized to the expression level of GAPDH. (A) Relative expression of DGK $\theta$ in diverse organs at E14.5. The values ( $m e a n \pm S E M, n=4)$ represent the relative level of expression in the whole brain compared with the lung, liver, intestine, or kidney. ${ }^{* *}$ indicates $P<0.01$ and ${ }^{*}$ indicates $P<0.05$ (B) Relative expression of DGK $\theta$ at each developmental stage in the epidermis of the dorsal skin. The amount of template for each PCR was normalized to the expression level of GAPDH. The values (mean \pm SEM, $n=4$ ) represent the relative level of expression at E14.5. * indicates $P<0.05$ compared with E17.5 or adulthood skin.

patterns indicated DGK $\theta$ protein was widely expressed in multiple organs and abundantly expressed in the brain and dorsal skin of mouse embryos. These results were confirmed by the analysis of DGK $\theta$ mRNA using RT-PCR and in situ hybridization (Figure 4 and Additional file 1: Figure S1B). The IHC results demonstrated that the expression of DGK $\theta$ significantly increased in the neuroepithelium surrounding the neural tube and ventricles in the brain. Prominent immunoreactivity of DGK $\theta$ was observed in a variety of neurons in the gestational brain over the period examined (E10.5-18.5). Especially, DGK $\theta$ was detected in the marginal zone of the neocortex, but not in the medial side of the lateral ventricle at E14.5 and E16.5. Since developing mammalian telencephalon is known to require atypical PKC [32], these results may suggest that DGK $\theta$ is associated with differentiation of the neuronal lineage or the locomotion of immature neurons [33]. During the prenatal period (E18.5), DGK $\theta$ was expressed in the developing cerebral cortex, hippocampus, and cerebellum. This distribution pattern is consistent with the in situ hybridization data for the adult rat brain [21]. Other DGK isoforms are known to be expressed in the postnatal brain [34-36] and are needed for various roles of mature neurons [37-39]. C. elegans DGK-1, which has $39 \%$ identity to mouse DGK $\theta$, regulates DAG levels generated by heterotrimeric G protein signaling in response to the neurotransmitters in nematode [40]. Genetic analysis of C.elegans, dgk-1 shows the role of dopamine controlled locomotion and serotonin-controlled egg-laying behavior [41]. The presence of DGK $\theta$ throughout the prenatal brain at E18.5 suggests either that DGK $\theta$ is involved in a common neuronal process, or that it provides a level of redundancy for other DGK isoforms in neurons $[29,39]$.

In the periphery, IHC results revealed that DGK $\theta$ is ubiquitously expressed in the layer of multiple organs during the embryonic period. In the intestine and kidney, the expression of DGK $\theta$ was prominent and persisted from E12.5 up to E18.5, while DGK $\theta$ expression in the lung, liver, and oropharyngeal membrane surrounding the tongue and nasal cavity was transient and attenuated before birth. Since DGK $\theta$ enhances the activation of EGF receptor stimulated with EGF via the counteraction of PKC activity in epidermoid cells [28], DGK $\theta$ may notably contribute to the development of epithelial cells during organogenesis (E10.5-E17.5). RTPCR analysis demonstrated that the DGK $\theta$ mRNA was expressed in abundance throughout the developmental process, with highest expression in multiple key organs. Thus, DGK $\theta$ may have persistent roles in diverse organs and tissues during the embryonic stages.

\section{Conclusions}

We revealed for the first time the distribution of DGK $\theta$ during the embryonic period. These results suggest that DGK $\theta$ may play important physiological roles not only in the brain, but also in diverse organs and tissues during the embryonic stages, and will serve as a basis for future in vivo genetic, functional, and mutational analyses of DGK $\theta$. 


\section{Methods \\ Animals}

Eight-week-old mice were purchased from Nippon SLC, Inc. (Hamamatsu, Japan). The animal works were reviewed and approved by the Care and Use of Laboratory Animals of Kobe University. All procedures using experimental animals were performed according to the Guidelines for the Care and Use of Laboratory Animals of Kobe University.

\section{Immunohistochemistry}

Most chemical reagents were purchased from Wako Pure Chemical Inc. (Oosaka, Japan). C57BL/6 J mice were anesthetized and transcardially perfused with cold PBS(-) and fixed in 4\% paraformaldehyde in PBS (-) for $10 \mathrm{~min}$. Mouse embryos were isolated from pregnant mice and fixed in $4 \%$ paraformaldehyde in PBS (-) overnight [42]. The mouse embryos were paraffin-embedded and sectioned at a $5 \mu \mathrm{m}$ thickness by Kyoudoubyouri, Inc. (Kobe, Japan) or Genostaff, Inc. (Tokyo, Japan). The serial paraffin sections were deparaffinized in xylene, and rehydrated using a graded series of ethanol from $99 \%$ to $50 \%$ and washed in distilled water. The sections were then incubated in $0.03 \% \mathrm{H}_{2} \mathrm{O}_{2}$ in PBS (-) for $1 \mathrm{hr}$, and permeabilized with $0.3 \%$ Triton $\mathrm{X}-100$ in PBS (-) for $30 \mathrm{~min}$. Subsequently, the sections were washed with $0.1 \%$ Tween 20 in PBS (-), and incubated in $0.1 \%$ Tween 20 in PBS (-) supplemented with goat serum (Dako, Glostrup, Denmark), serving as blocking reagent, for $1 \mathrm{hr}$. Next, the sections were treated with an anti-DGK $\theta$ antibody diluted (antibody \#1, 1:50 ; antibody \#2, 1:100) in Can Get Signal immunostain solution A (Toyobo, Tokyo, Japan) overnight. The antibodies against DGK $\theta$, antibody \#1 (immunogen of 691-820 aa) and antibody \#2 (immunogen of 677-883 aa), were purchased from Santa Cruz Biotechnology (California, USA), and BD Biosciences (California, USA), respectively. The control samples were probed with antibody that had been preincubated with a DGK $\theta$ blocking polypeptide. The bound antibodies were visualized using EnVision + System-HRP (Dako). The stained sections were dehydrated using a graded series of ethanol and mounted using a coverslip and Mount-Quick (Daido Sangyo, Gunma, Japan). The specimens were then photographed using an All-In-One Microscope system (BZ-8000 and BZ9000, Keyence, Japan), as reported previously [43].

The specific blocking polypeptide of DGK $\theta$ (677-883 aa) was generated by PCR using the following primers, with pEGFP-N3-DGK $\theta$ as template: 5' -AGTCGGAT CCGGCACAGGGAATGACCTTG-3' and 5'-CGAGTC ACGCTCCTCAAGTGATGAGGATCCAGTG-3'. The amplified PCR fragment was subcloned into the pQE30 vector (Qiagen, Tokyo, Japan) for expression and purification from $E$. coli.

\section{Reverse transcription-PCR analysis}

Reverse transcription-PCR (RT-PCR) was performed as described previously [44]. Briefly, organs or tissues were dissected from 15 embryos of mice under a stereomicroscope and frozen immediately on dry ice. The total RNA was isolated using a High Pure RNA Tissue Kit (Roche, Mannheim, Germany). For each tissue, equal amounts of RNA were reverse-transcribed with ReverTra Ace (Toyobo). The resulting cDNA was appropriately diluted and used to perform PCR with KOD-FX Neo (Toyobo).

The primer pair for mouse DGK $\theta$ was purchased from Takara-Bio Inc. (Otsu, Japan) and used to amplify the specific sequence : 5'-GGTTCAGAACAGGGCCAG GTAG-3' and 5'-AGGGCTGTAGGCAGGCAAAC-3' (NCBI : NM_199011, nt.3577-3706). RT-PCR was performed according to the manufacture's specification. Reactions for DGK $\theta$ were performed for 35 cycles at $98^{\circ} \mathrm{C}$ for $10 \mathrm{~s}, 60^{\circ} \mathrm{C}$ for $30 \mathrm{~s}$, and $68^{\circ} \mathrm{C}$ for $1 \mathrm{~min}$. After electrophoretic separation, the amplified fragments were quantified using the image-analysis software Image J.

\section{Additional files}

\begin{abstract}
Additional file 1: Figure S1. Conformation of DGK $\theta$ expression patterns by in situ hybridization and western blotting. (A) Highmagnification images showing the cardiac end of the stomach, and dorsal skin at E17.5. Epithelium (ep), dorsal skin (ds). (B) Sagittal sections showing the DGK $\theta$ mRNA expression by in situ hybridization analysis at E17.5. In situ hybridization were subjected to general method with digoxigenin (DIG)-labeled RNA antisense probe and visualized by anti-DIG antibody conjugated with alkaline phosphatase and the substrate NBT/BCIP (Roche). The specific probe (541-967 bp: the length $427 \mathrm{bp}$ ) were generated by DIG RNA Labeling kit [T7 RNA polymerase] using purified mouse DGK $\theta$ PCR product (541-967 bp: the length $427 \mathrm{bp}$ ) which produced by the following primers and mouse whole brain cDNA library : 5'-AGGGAGGGGAACCTGCCTTC-3' and 5'-GATCGAATTCTAATACGACTCACTATAGGGCCTCATTCCGA GCCAGGCGGG-3'. The deparaffinized sections were incubated for $16 \mathrm{hr}$ at $42^{\circ} \mathrm{C}$ in $4 \times$ SSC containing $40 \%$ formamide, $0.1 \times$ Denhardt's solution, $10 \mathrm{mM}$ DTT, and DIG-labeled RNA antisense probe $(1 \mu \mathrm{g} / \mathrm{ml})$. After hybridization, the sections were washed twice for $15 \mathrm{~min}$ at $37^{\circ} \mathrm{C}$ with $2 \times \mathrm{SSC}$ and subsequently washed twice for 5 min at $37^{\circ} \mathrm{C}$ with $0.4 \times$ SSC. (C) Estimation of the specificity of the anti-DGK $\theta$ antibodies. Adult mouse heart and whole brain were lysed in RIPA buffer containing protease inhibitor cocktails (Nacalai tesque). Total cell lysate (30 $\mu \mathrm{g}$ protein) was applied to SDS-polyacrylamide gel electrophoresis (7.5\%) and subjected to western blotting with anti-DGK $\theta$ antibody diluted (antibody \#1, 1:100; antibody \#2, 1:200) in Can Get Signal solution (Toyobo). The numbers on the right margin represent the molecular sizes of the pre-stained protein standard.
\end{abstract}

Additional file 2: Figure S2. Specificity of the anti-DGK $\theta$ antibody against DGK $\theta$ and other DGKs. EGFP-fused DGK $[45]$ and DGK 3 [13] were transiently expressed in HeLa cells and cultured for $24 \mathrm{hr}$. The cells were fixed with $4 \%$ paraformaldehyde in PBS (-) and permeabilized with $0.3 \%$ Triton X -100 in PBS $(-)$. Immunofluorescence staining was performed using the indicated antibodies followed by usual method [46]. Scale bar, $20 \mu \mathrm{m}$.

Additional file 3: Figure S3. Expression patterns of DGK $\theta$ at E17.5. (A) High-magnification images showing the area of the renal medulla at E17.5. Collecting tubule (ct), Bowman's capsule (bc). (B-H) Sagittal sections showing the staining pattern of anti-DGK $\theta$ antibody in abdomen and head area. Stomach (st), pancreas (pa), kidney (ki), duodenum (du), liver (li), neocortex (nx). 


\section{Competing interests}

All authors of this paper declare no conflict of interest.

\section{Authors' contributions}

SU designed the experiments and analyzed the data. SU wrote the manuscript. BT provided good comments and helped to write the manuscript. All authors read and approved the final manuscript.

\section{Acknowledgment}

This investigation was supported by Grants-in-aid for Scientific Research on Priority Areas from the Ministry of Education, Culture, Sports, Science and Technology of Japan.

\section{Author details}

'Department of Agrobioscience, Graduate School of Agricultural Science, Kobe University, Kobe, Hyogo, Japan. Department of Biological Chemistry, Johns Hopkins University School of Medicine, Baltimore, Maryland, USA.

Received: 7 February 2013 Accepted: 27 September 2013 Published: 1 October 2013

\section{References}

1. Ahmed S, Lee J, Kozma R, Best A, Monfries C, Lim L: A novel functional target for tumor-promoting phorbol esters and lysophosphatidic acid. The p21rac-GTPase activating protein n-chimaerin. J Biol Chem 1993, 268(15):10709-10712.

2. Jones DR, Sanjuan MA, Stone JC, Merida I: Expression of a catalytically inactive form of diacylglycerol kinase alpha induces sustained signaling through RasGRP. FASEB J 2002, 16(6):595-597.

3. Topham MK, Prescott SM: Diacylglycerol kinase zeta regulates Ras activation by a novel mechanism. J Cell Biol 2001, 152(6):1135-1143.

4. Venkatachalam K, Zheng F, Gill DL: Regulation of canonical transient receptor potential (TRPC) channel function by diacylglycerol and protein kinase C. J Biol Chem 2003, 278(31):29031-29040.

5. Nishizuka $Y$ : Protein kinase $C$ and lipid signaling for sustained cellular responses. FASEB J 1995, 9(7):484-496.

6. Yang C, Kazanietz MG: Divergence and complexities in DAG signaling: looking beyond PKC. Trends Pharmacol Sci 2003, 24(11):602-608.

7. Limatola C, Schaap D, Moolenaar WH, van Blitterswijk WJ: Phosphatidic acid activation of protein kinase C-zeta overexpressed in COS cells: comparison with other protein kinase $C$ isotypes and other acidic lipids. Biochem J 1994, 304(Pt 3):1001-1008.

8. Topham MK, Prescott SM: Mammalian diacylglycerol kinases, a family of lipid kinases with signaling functions. J Biol Chem 1999, 274(17):11447-11450.

9. Kanoh H, Yamada K, Sakane F: Diacylglycerol kinases: emerging downstream regulators in cell signaling systems. Can J Biochem 2002, 131(5):629-633.

10. Merida I, Avila-Flores A, Merino E: Diacylglycerol kinases: at the hub of cell signalling. Biochem J 2008, 409(1):1-18.

11. van Blitterswijk WJ, Houssa B: Properties and functions of diacylglycerol kinases. Cell Signal 2000, 12(9-10):595-605.

12. Wattenberg BW, Pitson SM, Raben DM: The sphingosine and diacylglycerol kinase superfamily of signaling kinases: localization as a key to signaling function. J Lipid Res 2006, 47(6):1128-1139.

13. Shirai Y, Kouzuki T, Kakefuda K, Moriguchi S, Oyagi A, Horie K, Morita SY, Shimazawa M, Fukunaga K, Takeda J, et al: Essential role of neuronenriched diacylglycerol kinase (DGK), DGKbeta in neurite spine formation, contributing to cognitive function. PLoS One 2010, 5(7):e11602.

14. Flores I, Casaseca T, Martinez AC, Kanoh H, Merida I: Phosphatidic acid generation through interleukin 2 (IL-2)-induced alpha-diacylglycerol kinase activation is an essential step in IL-2-mediated lymphocyte proliferation. J Biol Chem 1996, 271(17):10334-10340.

15. Olenchock BA, Guo R, Carpenter JH, Jordan M, Topham MK, Koretzky GA Zhong XP: Disruption of diacylglycerol metabolism impairs the induction of T cell anergy. Nat Immunol 2006, 7(11):1174-1181.

16. Formoso D, Weber RN, Atkins MS: Gentrification and urban children's wellbeing: tipping the scales from problems to promise. Am J Community Psychol 2010, 46(3-4):395-412.
17. Liu CH, Machado FS, Guo R, Nichols KE, Burks AW, Aliberti JC, Zhong XP: Diacylglycerol kinase zeta regulates microbial recognition and host resistance to Toxoplasma gondii. J Exp Med 2007, 204(4):781-792.

18. Rodriguez De Turco EB, Tang W, Topham MK, Sakane F, Marcheselli VL, Chen C, Taketomi A, Prescott SM, Bazan NG: Diacylglycerol kinase epsilon regulates seizure susceptibility and long-term potentiation through arachidonoyl- inositol lipid signaling. Proc Natl Acad Sci USA 2001, 98(8):4740-4745.

19. Hozumi Y, Goto K: Diacylglycerol kinase beta in neurons: functional implications at the synapse and in disease. Adv Biol Regul 2012, 52(2):315-325

20. McMullan R, Hiley E, Morrison P, Nurrish SJ: Rho is a presynaptic activator of neurotransmitter release at pre-existing synapses in C-elegans. Gene Dev 2006, 20(1):65-76.

21. Houssa B, Schaap D, van der Wal J, Goto K, Kondo H, Yamakawa A, Shibata M, Takenawa T, van Blitterswijk WJ: Cloning of a novel human diacylglycerol kinase (DGKtheta) containing three cysteine-rich domains, a proline-rich region, and a pleckstrin homology domain with an overlapping Ras-associating domain. J Biol Chem 1997, 272(16):10422-10428.

22. Tabellini G, Bortul R, Santi S, Riccio M, Baldini G, Cappellini A, Billi AM, Berezney R, Ruggeri A, Cocco L, et al: Diacylglycerol kinase-theta is localized in the speckle domains of the nucleus. Exp Cell Res 2003, 287(1):143-154.

23. Houssa B, de Widt J, Kranenburg O, Moolenaar WH, van Blitterswijk WJ: Diacylglycerol kinase theta binds to and is negatively regulated by active RhoA. J Biol Chem 1999, 274(11):6820-6822.

24. van Baal J, de Widt J, Divecha N, van Blitterswijk WJ: Translocation of diacylglycerol kinase theta from cytosol to plasma membrane in response to activation of $\mathrm{G}$ protein-coupled receptors and protein kinase C. J Biol Chem 2005, 280(11):9870-9878.

25. Tu-Sekine B, Goldschmidt H, Petro E, Raben DM: Diacylglycerol kinase theta: Regulation and stability. Adv Biol Regul 2013, 53(1):118-126.

26. Tu-Sekine B, Raben DM: Dual Regulation of Diacylglycerol Kinase (DGK)theta: POLYBASIC PROTEINS PROMOTE ACTIVATION BY PHOSPHOLIPIDS AND INCREASE SUBSTRATE AFFINITY. J Biol Chem 2012,

287(50):41619-41627.

27. Tu-Sekine B, Raben DM: Regulation of DGK-theta. J Cell Physiol 2009, 220(3):548-552.

28. van Baal J, de Widt J, Divecha N, van Blitterswijk WJ: Diacylglycerol kinase theta counteracts protein kinase C-mediated inactivation of the EGF receptor. Int J Biochem Cell Biol 2012, 44(11):1791-1799.

29. Tabellini G, Billi AM, Fala F, Cappellini A, Evagelisti C, Manzoli L, Cocco L, Martelli AM: Nuclear diacylglycerol kinase-theta is activated in response to nerve growth factor stimulation of PC12 cells. Cell Signal 2004, 16(11):1263-1271.

30. Tu-Sekine B, Ostroski M, Raben DM: Modulation of diacylglycerol kinase theta activity by alpha-thrombin and phospholipids. Biochemistry 2007 46(3):924-932.

31. Baldanzi G, Alchera E, Imarisio C, Gaggianesi M, Dal Ponte C, Nitti M, Domenicotti C, van Blitterswijk WJ, Albano E, Graziani A, et al: Negative regulation of diacylglycerol kinase theta mediates adenosine-dependent hepatocyte preconditioning. Cell Death Differ 2010, 17(6):1059-1068.

32. Imai F, Hirai S, Akimoto K, Koyama H, Miyata T, Ogawa M, Noguchi S, Sasaoka T, Noda T, Ohno S: Inactivation of aPKClambda results in the loss of adherens junctions in neuroepithelial cells without affecting neurogenesis in mouse neocortex. Development 2006, 133(9):1735-1744.

33. Sekine K, Honda T, Kawauchi T, Kubo K, Nakajima K: The outermost region of the developing cortical plate is crucial for both the switch of the radial migration mode and the Dab1-dependent "inside-out" lamination in the neocortex. J Neurosci 2011, 31(25):9426-9439.

34. Adachi N, Oyasu M, Taniguchi T, Yamaguchi Y, Takenaka R, Shirai Y, Saito N: Immunocytochemical localization of a neuron-specific diacylglycerol kinase beta and gamma in the developing rat brain. Brain Res Mol Brain Re 2005, 139(2):288-299.

35. Goto K, Funayama M, Kondo $\mathrm{H}$ : Cloning and expression of a cytoskeletonassociated diacylglycerol kinase that is dominantly expressed in cerebellum. Proc Natl Acad Sci USA 1994, 91(26):13042-13046.

36. Goto K, Kondo H: Molecular cloning and expression of a 90-kDa diacylglycerol kinase that predominantly localizes in neurons. Proc Natl Acad Sci USA 1993, 90(16):7598-7602. 
37. Goto K, Kondo H: Diacylglycerol kinase in the central nervous systemmolecular heterogeneity and gene expression. Chem Phys Lipids 1999, 98(1-2):109-117

38. Musto A, Bazan NG: Diacylglycerol kinase epsilon modulates rapid kindling epileptogenesis. Epilepsia 2006, 47(2):267-276.

39. Tu-Sekine B, Raben DM: Regulation and roles of neuronal diacylglycerol kinases: a lipid perspective. Crit Rev Biochem Mol Biol 2011, 46(5):353-364

40. Nurrish S, Segalat L, Kaplan JM: Serotonin inhibition of synaptic transmission: Galpha(0) decreases the abundance of UNC-13 at release sites. Neuron 1999, 24(1):231-242.

41. Miller KG, Emerson MD, Rand JB: Goalpha and diacylglycerol kinase negatively regulate the Gqalpha pathway in C. elegans. Neuron 1999, 24(2):323-333.

42. Bilasy SE, Satoh T, Ueda S, Wei P, Kanemura H, Aiba A, Terashima T, Kataoka T: Dorsal telencephalon-specific RA-GEF-1 knockout mice develop heterotopic cortical mass and commissural fiber defect. Suppl Eur J Neurosci 2009, 29(10):1994-2008.

43. Kanemura H, Satoh T, Bilasy SE, Ueda S, Hirashima M, Kataoka T: Impaired vascular development in the yolk sac and allantois in mice lacking RAGEF-1. Biochem Biophys Res Commun 2009, 387(4):754-759.

44. Ueda S, Kitazawa S, Ishida K, Nishikawa Y, Matsui M, Matsumoto H, Aoki T, Nozaki S, Takeda T, Tamori Y, et al: Crucial role of the small GTPase Rac1 in insulin-stimulated translocation of glucose transporter 4 to the mouse skeletal muscle sarcolemma. FASEB J 2010, 24(7):2254-2261.

45. Tu-Sekine B, Raben DM: Characterization of cellular DGK-theta. Adv Enzyme Regul 2010, 50(1):81-94.

46. Ueda S, Kataoka T, Satoh T: Role of the Sec14-like domain of Dbl family exchange factors in the regulation of Rho family GTPases in different subcellular sites. Cell Signal 2004, 16(8):899-906.

doi:10.1186/1471-213X-13-35

Cite this article as: Ueda et al:: The expression of diacylglycerol kinase theta during the organogenesis of mouse embryos. BMC Developmental Biology 2013 13:35.

\section{Submit your next manuscript to BioMed Central and take full advantage of:}

- Convenient online submission

- Thorough peer review

- No space constraints or color figure charges

- Immediate publication on acceptance

- Inclusion in PubMed, CAS, Scopus and Google Scholar

- Research which is freely available for redistribution 